\title{
PERSPECTIVAS TEÓRICAS SOBRE DESIGUALDAD(ES) SOCIAL(ES): aportes para el análisis de las políticas compensatorias y socioeducativas en Argentina
}

\author{
Nicolás Carriquiriborde ${ }^{1}$
}

\begin{abstract}
RESUMEN
El presente artículo presenta un recorrido histórico y conceptual sobre las teorías que abordan el problema de la desigualdad social en las sociedades modernas y contemporáneas para proponer luego un análisis de cuatro estudios vinculados a políticas públicas que constituyen los antecedentes de una investigación en curso en la que se indaga el trabajo de enseñar de profesores/as de Educación Física en políticas destinadas al tratamiento de la desigualdad social y educativa en Argentina, particularmente las políticas socioeducativas. El trabajo permitirá, por un lado, observar cómo la problemática de la desigualdad en las ciencias sociales ha pasado de una concepción unidimensional de la desigualdad social, con explicaciones economicistas o culturalistas, hacia posiciones y abordajes multidimensionales que intentan captar el fenómeno social incluyendo dimensiones culturales, sociales, económicas, articulando diferentes niveles. Por otro lado, sitúa y da cuenta del surgimiento de un novedoso modo de tratamiento de la desigualdad social en Argentina, a través de políticas estatales particulares, las llamadas políticas compensatorias de fin de siglo XX y las denominadas socioeducativas en las primeras décadas del siglo XXI. Con ello se presentan los principales antecedentes de investigación sobre este tipo de políticas y se los analiza desde aquellas perspectivas sociológicas. El análisis de los trabajos da cuenta de enfoques anclados en una perspectiva macroestructural con énfasis en la dimensión económica de la desigualdad social y otros que incluyen la dimensión macro estructural, pero enfatizando el nivel de las interacciones institucionales y mediaciones locales.
\end{abstract}

Palabras clave: Desigualdad social. Políticas públicas. Argentina.

\section{Perspectivas teóricas sobre as desigualdades sociais: contribuições para a análise de políticas compensatórias e socioeducativas na Argentina}

\begin{abstract}
RESUMO
Este artigo apresenta uma revisão histórica e conceitual das teorias que abordam 0 problema da desigualdade social nas sociedades modernas e contemporâneas para propor, em seguida, uma análise de quatro estudos vinculados a políticas públicas que constituem o pano de fundo de uma investigação em andamento que investiga o trabalho de professores de Educação Física em políticas destinadas ao tratamento da desigualdade social e educacional na Argentina, particularmente políticas socioeducativas. O trabalho permitirá, por um lado, observar como o problema da desigualdade nas ciências sociais passou de uma concepção unidimensional da desigualdade social, com explicações

\footnotetext{
1 Maestrando en Educación. Profesor en Educación Física. Facultad de Humanidades y Ciencias de la Educación. Universidad Nacional de La Plata. Centro Interdisciplinario Cuerpo, Educación y Sociedad. Instituto de Investigaciones en Humanidades y Ciencias Sociales/CONICET. La Plata, Buenos Aires, Argentina. Orcid iD: https://orcid.org/0000-00033656-7984. E-mail: nicolas.carriquiriborde@gmail.com
} 
econômicas ou culturalistas, para posições e abordagens multidimensionais que tentam capturar o fenômeno social, incluindo dimensões culturais, sociais e econômicas, articulando diferentes níveis. Por outro lado, localiza e explica o surgimento de uma nova maneira de tratar a desigualdade social na Argentina, por meio de políticas estaduais específicas, as chamadas políticas compensatórias do final do século XX e as chamadas políticas socioeducativas nas primeiras décadas do século XXI. Com isso, os principais antecedentes de pesquisas sobre esse tipo de política são apresentados e analisados a partir dessas perspectivas sociológicas. A análise dos trabalhos mostra abordagens ancoradas em uma perspectiva macroestrutural, com ênfase na dimensão econômica da desigualdade social e outras que incluem a dimensão macroestrutural, mas enfatizando o nível de interações institucionais e mediações locais.

Palavras-chave: Desigualdade Social. Políticas públicas. Argentina

\title{
Theoretical perspectives on social inequality (s): contributions for the analysis of compensatory and socio-educational policies in Argentina
}

\begin{abstract}
This article presents a historical and conceptual overview of the theories that address the problem of social inequality in modern and contemporary societies, and then propose an analysis of four studies related to public policies that constitute the background to ongoing research in which investigates the work of teaching of Physical Education teachers in policies aimed at treating social and educational inequality in Argentina, particularly socioeducational policies. The work will allow, on the one hand, to observe how the problem of inequality in the social sciences has gone from a one-dimensional conception of social inequality, with economistic or culturalist explanations, to multidimensional positions and approaches that try to capture the social phenomenon including cultural dimensions, social, economic, articulating different levels. On the other hand, it locates and accounts for the emergence of a novel way of treating social inequality in Argentina, through particular state policies, the so-called compensatory policies of the end of the 20th century and the socalled socio-educational policies in the first decades of the 21 st century. With this, the main antecedents of research on this type of policy are presented and analyzed from those sociological perspectives. The analysis shows approaches to a structural macro perspective with emphasis on the economic dimension of social inequality and others that include the macro structural dimension, but emphasizing the level of institutional interactions and local mediations.
\end{abstract}

Keywords: Social inequality. Public policies. Argentina.

\section{INTRODUCCIÓN}

Los estudios sobre la desigualdad social y la forma en que ésta se produce y reproduce, ha sido objeto de diversas aproximaciones en las ciencias sociales. Tales perspectivas han arrojado luz sobre algunos aspectos particulares del fenómeno de las desigualdades: hay desarrollos teóricos que enfocan su mirada en las experiencias de los individuos, otros más centrados en las pautas de relaciones e intercambios interinstitucionales desiguales y 
aquellos que priorizan el enfoque en las condiciones estructurales asimétricas que se imponen a los sujetos.

La desigualdad social o el problema de las desigualdades sociales como campo de estudio debemos situarlo a partir de la Modernidad. La misma implicó serias y grandes transformaciones políticas, económicas y culturales con respecto a las sociedades tradicionales (Bendix, 1974). En el plano político significó la instauración de sistemas sostenidos en el principio de la soberanía de la población; en lo económico implicó la progresiva instauración del modo de producción capitalista; en lo social se abandonó el orden adscripto que definía las posiciones de acuerdo al origen social para hacer del mérito el criterio que legitima los lugares que se ocupan en la sociedad (Hobsbawn, 1964). Al mismo tiempo, se propone una sociedad de iguales ante la ley y la participación en el orden político.

La combinación de un sistema político que produce un individuo libre, soberano de sí mismo, igual ante la ley, junto a un modelo de producción que habilita lo que Hobsbawn (1964) llamo carrera abierta al talento produce una nueva sociedad con ganadores y perdedores. Esta sociedad al romper las cadenas que ataban a los individuos a los estamentos de origen y convertirlos en ciudadanos libres, libra la competencia por la subsistencia a sus talentos individuales. La Modernidad, entonces, instala la inevitable e inacabable tensión entre igualdad y libertad y es en relación a estas coordenadas conceptuales que el problema de la desigualdad social será abarcado.

\section{La desigualdad como problema de estudio}

Mora (2004) ubica aquella discusión, entre la ponderación de la igualdad y la ponderación de la liberad, en los tiempos de la llustración, más precisamente, en las obras de Hobbes y Rousseau. Según la autora, ambos constituyen e inauguran dos corrientes de pensamientos: la corriente liberal referenciada en Hobbes y la corriente crítica que identifica con Rousseau. Ambas abordarán la problemática de la desigualdad social a partir de la 
pregunta por su origen, es decir, por cómo es la relación individuo y sociedad y por los potenciales efectos del tratamiento en el marco de esas concepciones.

Para la autora mencionada, la corriente liberal se caracteriza por la importancia declarada a la libertad por sobre la igualdad. La igualdad para esta corriente refiere a una igualdad política, no necesariamente social. Su mirada se centra en la libertad de los individuos dotados naturalmente de capacidades y talentos para desarrollarse en sociedad. Por ello, es fundamental garantizar la igualdad de oportunidades para que las capacidades, habilidades y talentos de los individuos en libre competencia definan sus lugares en la sociedad. De este modo, la desigualdad social se auto explica por la desigual asignación natural de talentos y se justifica en la competencia a la que fueron sometidos esos talentos.

Por su parte, la corriente crítica enfatiza la importancia asignada a la igualdad. Realiza una crítica de aquella concepción que pondera al individuo por sobre la comunidad oponiendo en su argumentación a la propiedad privada como productora de las desigualdades y no a la distribución natural de talentos. La mirada está puesta en la estructura social y se prioriza el derecho colectivo por sobre el individual. Con todo, la crítica radical que realiza esta corriente refiere al carácter histórico y social de la producción de desigualdad y no a su condición natural.

Así, podemos ver que la discusión entre la corriente liberal y la corriente crítica expresa un debate profundo y transversal a las ciencias sociales que refiere al de la igualdad y la libertad. En la década de 1970, para Mora (2004) hay una ruptura en el campo de los estudios sobre desigualdades. Para ella, "la confluencia de nuevos desarrollos teóricos, en conjunto con la existencia de nuevas realidades sociales, que no se podían explicar con base en los esquemas clásicos, sirvieron como acicates para la redefinición del debate en el campo de nuestro interés." (p.14) La desigualdad social ya no es posible explicarla, abordarla, tematizarla en los términos clásicos (más vinculada a posiciones filosóficas e ideológicas) sino que es preciso analizarla 
a partir de marcos conceptuales que den cuenta del carácter multidimensional, relacional y dinámico de las (ahora en plural) desigualdades sociales.

Recuperando a Turner (1988 citado en Mora, 2004) la autora afirma que se pasa de un concepto filosófico de la igualdad a uno sociológico destacando cuatro dimensiones de la igualdad:

$>\quad$ igualdad ontológica: refiere a un argumento moral sobre la igualdad que puede tener un carácter religioso (todos los seres humanos son iguales ante Dios) o político (en la teoría marxista el hombre es universalmente libre);

$>$ igualdad de oportunidades: acceso a la estructura de oportunidades. Es un sistema que se justifica en la competencia de los talentos individuales y el esfuerzo personal. Se trata de igualar posibilidades en tanto se legitiman desigualdades de resultados;

> igualdad de condiciones: para que todos/as los/as individuos de una sociedad tengan acceso a las mismas oportunidades es necesario nivelar las condiciones sociales de acceso a esas oportunidades. Es una igualdad en el acceso y puede ser complementaria a la igualdad de oportunidades, en tanto primero se debe garantizar el acceso por igual a todos/as para lvego habilitar la competencia de oportunidades;

$>$ igualdad de resultados: que expresaría el máximo grado de igualdad. Refiere a transformar las desigualdades sociales de origen en igualdades al final del proceso.

Otra teoría que aparece con fuerza en el debate sobre la desigualdad en esta década refiere a la desarrollada por Rawls (1973 citado en Mora, 2004) en la que la autora considera "orientada a resolver la dicotomía libertad-igualdad que caracteriza el debate clásico sobre la desigualdad social" (2014, p. 30). En ella se sostiene que no es posible la igualdad en el capitalismo, por tanto, la pregunta ronda en torno a cómo reducir la desigualdad y cuáles serían los grados de desigualdad inaceptables para 
una sociedad. En palabras de Mora: "una sociedad que, aceptando la existencia de desigualdades entre sus miembros, crea las condiciones institucionales, políticas, sociales y económicas indispensables para que cada uno de sus integrantes tenga garantizado un nivel de integración y bienestar social que le permita ser un miembro activo de la comunidad" (2004: p. 31).

Para Rawls, las sociedades tienen que crear instituciones que permitan la integración social. Propone un abordaje procedimental de la justicia para reglas de asignación de recompensas que aseguren que el resultado distributivo será justo. Su hipótesis o recurso metodológico, es que existe una posición original expresada en un contrato social a partir del cual se sentarían las bases para juzgar los grados moralmente aceptables de desigualdad y con ello operativizar acciones y políticas con una noción de justicia redistributiva. Así, es evidente que para Rawls "Ia pregunta ya no es cómo abolir la desigualdad social, sino, más bien, cómo construir una sociedad más justa, basada en un consenso social amplio y legítimo" (Mora, 2004: p. 31).

Ubicándonos ya en los abordajes contemporáneos sobre desigualdades sociales, François Dubet (2011) retoma el debate entre libertad e igualdad y lo hace analizando las potencialidades, efectos y debilidades de dos grandes modelos de igualdad social que han caracterizado a las sociedades occidentales, particularmente a la francesa: la igualdad de posiciones y la igualdad de oportunidades.

La igualdad de posiciones para Dubet es un modelo de igualdad que se establece en relación a la estructura de clases, es decir en la pareja explotador/explotado. El acceso a un empleo estable y, por lo tanto, a un ingreso implica acceder a una posición social de la cual se parte $y$, por tanto, otorga al ciudadano un lugar en la escala social. Este modelo es deudor de los Estados de Bienestar caracterizados por una alta tasa de empleo y de beneficios sociales para una gran porción de la sociedad. 
El Estado aquí es el garante de tales posiciones y establece una relación directa con aquellos que accedieron a dichas posiciones y por tanto produce un debilitamiento en las relaciones entre "clasados", en la medida en que él mismo se torna asegurador de las posiciones sociales. En este sentido, se afirma que la igualdad de posiciones es conservadora al ignorar otros elementos de la dinámica social que exceden a la cuestión del trabajo. Al querer garantizar la igualdad de posiciones por medio de la generación de empleos ignora desigualdades que provienen de otros mecanismos, como el caso de la desigualdad de género, las desigualdades étnicas, raciales, entre otras. Para Dubet:

La igualdad de las posiciones está asociada a una representación de la sociedad en términos de estratificación socio profesional y de clases sociales: las posiciones se definen con independencia de quienes las ocupen. Por el contrario, la igualdad de las oportunidades conduce a definir grupos sociales en términos de discriminaciones y de desventajas, es decir, en función de sus chances objetivas de acceder

a todas las posiciones. (201 1: p. 58)

La igualdad de oportunidades es para Dubet (2011), el criterio de origen del proyecto Moderno. Fue el modelo que permitió distribuir y justificar sujetos formalmente iguales en posiciones sociales desiguales. Es el criterio de justicia dominante en las sociedades democráticas y es el que se utiliza para anular el criterio de la herencia o el azar. El concepto de igualdad de oportunidades queda asociado al modelo de las elites dirigentes que han visto en él el acceso, vía mérito individual, a posiciones sociales elevadas. Vuelve a la escena principal cada vez que el modelo de la igualdad de posiciones se deteriora o cuando este ignora otras desigualdades como las vinculadas al género, la inmigración, la raza. Por ello, este modelo ya no se asienta en la estructura de clases, en el tándem explotación-trabajo sino en el de la discriminación-identidad. 
La igualdad de oportunidades potencia hasta el hartazgo la iniciativa individual al crear la ficción de libre competencia en igualdad de condiciones. Establece un piso de igualdad (todos son iguales ante la ley) para el acceso a bienes sociales donde cada sujeto debe valerse por sí mismo. Las fronteras sociales ceden ante las fronteras culturales y se crea así todo un universo moral en torno a las figuras de vencedores y vencidos.

Frente a estos dos modelos, el autor se inclina por el de la igualdad de posiciones, por un lado, porque considera que crea un sistema de derechos y de obligaciones que contribuye a resaltar aquello del orden de lo común contribuyendo a reforzar lazos de solidaridad. Y por otro, porque sería la mejor manera de llevar adelante la igualdad de oportunidades, en tanto considera que una sociedad donde las posiciones sociales son más parejas permite que la igualdad de oportunidades se realice sobre la base de esos mayores niveles de igualdad social.

Otro autor contemporáneo que se pregunta por el origen de las desigualdades es Reygadas (2004). En su artículo se explicitan tres formas, tres modos, de responder por la cuestión de la desigualdad considerando que ellas, por separadas, no otorgan una respuesta suficiente en torno a la producción de desigualdades. Esto es así porque para el autor la desigualdad social es un problema multidimensional y por tanto no es posible abordarla solo desde un enfoque $\mathrm{o}$, mejor, las respuestas posibles pueden arrojarnos luces sobre algunos de sus aspectos y mantener en la oscuridad otros tantos.

Para Reygadas existen tres grandes teorías sobre la producción de desigualdad:

Las teorías individualistas han puesto el acento en la distribución de capacidades y recursos entre los agentes, las teorías interaccionistas han hecho énfasis en las pautas de relaciones y en los intercambios desiguales y, a su vez, las teorías holísticas se han concentrado en las características asimétricas de las estructuras sociales. (2004: p. 7) [el destacado me pertenece] 
Las teorías individualistas tienen la potencialidad de dar cuenta de los usos y capacidades que individuos despliegan y/o acumulan en la dinámica social, como los bienes materiales y recursos económicos, las capacidades cognitivas, afectivas y relacionales que evidencian individuos en determinado ámbito y permiten su movilidad y distancia social. También aquellos aspectos interiorizados o difíciles de captar en relación a valoraciones culturales, de prestigio, condición de género, etnia, nacionalidad, y dispositivos más sutiles de diferenciación que producen desigualdades.

Como contrapartida, al decir de Reygadas (2004), estas teorías presentan una serie de problemas: no dan cuenta del origen social de los atributos individuales, las valoraciones a esos atributos son socialmente construidas y no universales o neutrales $y$, al evidenciar una concepción individualista de lo social, no pueden explicar de forma cabal los procesos de expropiación, es decir, los procesos de explotación y acaparamiento de oportunidades, a los que buena parte de los individuos se ven sometidos.

Para las teorías interaccionistas, nos interesa destacar lo que el autor denomina como "Ias principales estrategias político-simbólicas que interviene en la producción de desigualdad" (2004: p. 15) y refieren, en primer término, a las que imputan características positivas al grupo social de pertenencia, es decir a las que presentan los privilegios de un grupo como si fuesen portadoras de rasgos especiales. En segundo lugar, y como complemento de estas, las que otorgan características negativas a otros grupos. Por otro lado, aquellas que tienden a preservar la separación entre agrupaciones conformadas, consolidando las distancias sociales. Y, por último, el trabajo de legitimación de intereses particulares como universales, es decir de naturalización de formas de desigualdad.

Estas desigualdades, para Reygadas (2004), se encuentran resguardadas por fronteras que separan a los grupos sociales. Las mismas toman forma de fronteras como barreras físicas, tales como muros, rejas, 
etcétera. Otras las caracteriza como dispositivos institucionales como prohibiciones, derechos, patentes y otras. Por último, fronteras que no se materializan en objetos o disposiciones institucionales sino más sutiles y simbólicas como las referidas a la imposibilidad de mujeres de acceder a mejores empleos que varones o igual remuneración que estos por igual trabajo (techo de cristal o glass ceiling), clasificaciones arbitrarias, estigmas y otros mecanismos similares.

Como se observa estas teorías nos permiten ver las desigualdades como producción de dinámicas ya no solo individuales, sino como parte de una compleja red de dispositivos $e$ instituciones que inciden en la constitución de ventajas y desventajas sociales.

Finalmente, el autor hará mención a las teorías holísticas que ponen su atención en las dinámicas macro estructurales propias del modelo actual de acumulación en fase de globalización y transnacionalización, donde propiedad privada y medios de producción se articulan y vinculan con la producción y circulación de conocimiento produciendo nuevas dinámicas de explotación y acaparamiento por parte de grupos sociales.

La desventaja de centrar la atención solo en los aspectos estructurales que producen desigualdad es que estas teorías no nos permiten dar cuenta de la capacidad de agencia de los individuos, es decir lo que esos sujetos y esas instituciones hacen con las condiciones de posibilidad que plantea la dinámica social.

Con todo, para Reygadas (2004), las desigualdades tienen que ver con relaciones de poder, y el poder tiene que ver con diferentes recursos y capacidades, por tanto, la desigualdad es un fenómeno complejo que presenta varios elementos, imposible de reducirla a uno solo. De ahí que el autor proponga un abordaje que contenga 0 de cuenta de las tres dimensiones.

Por su parte, Saraví va a plantear el problema de la desigualdad social en términos de fragmentación social, a la que caracteriza como la 
producción de "mundos social y culturalmente distantes y aislados uno de otros" (2015: p. 27). Para el autor, la reconfiguración que llevo adelante el modelo económico mundial en las últimas dos décadas del siglo XX, particularmente en Latinoamérica, puso en el centro de la cuestión el problema de la exclusión social. Esta hizo evidente la multidimensionalidad de "la acumulación de desventajas sociales, económicas, políticas y simbólicas en los procesos de ‘exclusión social’” (2015, p. 33).

Sin involucrarnos en su desarrollo sobre la cuestión de la subjetividad, procesos de subjetivización y experiencias de desigualdad, debido a los objetivos y extensión propia del presente artículo, podemos ceñirnos a su planteo conceptual de exclusiones recíprocas e inclusiones desiguales. Para el autor ya no es posible abordar las desigualdades sociales sosteniendo un principio apriorístico, como podría ser el de clase social, sino que, producto de las nuevas dinámicas sociales y procesos de acumulación de desventajas (y ventajas), es necesario articular ese análisis a las dimensiones socioculturales que producen y reproducen circuitos diferenciados, distanciados y fragmentados de desventajas y privilegios. Las exclusiones recíprocas aluden a estos distanciamientos entre sectores, marcados por el acaparamiento y privación de privilegios de unos por sobre otros y su inclusión diferenciada que los sitúa en grupos y dinámicas propias.

Como es posible observar, los estudios sobre desigualdad social han pasado de posiciones y abordajes de tipo filosóficos hacia perspectivas sociológicas con énfasis diferentes según se prioricen dinámicas económicas, culturales, políticas y abarquen diferentes niveles: micro-niveles (centrados en los individuos), meso-niveles (instituciones e interacciones entre grupos) y macro-niveles (dinámicas globales, transnacionales). Con ello, se ha pasado de una concepción unidimensional de la desigualdad social, con explicaciones economicistas o culturalistas, hacia posiciones y abordajes multidimensionales que intentan captar el fenómeno social incluyendo dimensiones culturales, sociales, económicas, articulando diferentes niveles. 


\section{Las políticas públicas para el abordaje de la desigualdad social}

Luego de este recorrido conceptual a partir de las principales perspectivas sociológicas en relación a la problemática de la desigualdad buscaremos valernos de sus herramientas para analizar algunos de los antecedentes de investigación relevados en una investigación en curso donde se analizan las posiciones docentes ${ }^{2}$ que profesores y profesoras de Educación Física (EF) asumen en un programa ${ }^{3}$ de política pública que tiene como mandatos la igualdad, calidad e inclusión social. En ella interesa indagar los sentidos que circulan en torno al trabajo de enseñar en profesores/as de EF en políticas socioeducativas ${ }^{4}$.

Si bien la unidad de análisis la constituyen los profesores y profesoras de Educación Física, la política socioeducativa en general, y el programa Centro de Actividades Infantiles (CAI) en particular, conforman el objeto de estudio en tanto nos interesa indagar, como se dijo, las posiciones docentes que asumen estos profesores y profesoras en el marco de una política orientada a la igualdad, calidad e inclusión. Por tanto, la dimensión de la política socioeducativa como discurso que interpela a profesores y profesoras en su trabajo de enseñar ${ }^{5}$ cobra relevancia en la búsqueda de poder captar los diversos elementos discursivos que se articulan de una particular manera conformando un tipo de posición docente.

Finalmente, presentaré una breve caracterización sobre este tipo particular de políticas estatales para luego analizar cuatro antecedentes de investigación sobre el tratamiento de la desigualdad social y educativa en las últimas décadas del siglo XX y primeras del XXI en Argentina, intentando

\footnotetext{
2 Ver Southwell y Vassiliades (2014) y Southwell (2017).

3 El programa refiere a los Centros de Actividades Infantiles (CAl) creado en el año 2010 dependiente de la Dirección Nacional de Políticas Socioeducativas (DNPS).

4 Para dar cuenta de esta multiplicidad de sentidos en relación a lo socioeducativo se puede consultar Carriquiriborde (2019). Disponible en:

https://seer.ufs.br/index.php/revtee/article/view/9451

5 Un estudio que indaga sobre identidades y representaciones docentes en el trabajo de enseñar lo constituye el de Correa et. al. "IDENTIDAD DOCENTE: representaciones de profesores/as en narrativas ribereñas del Rio Quianduba en Abaetetuba-Pa" Revista Exitus, Santarém/PA, v. 10, 2020, p. 1-28.
} 
dar cuenta los diversos enfoques teóricos sobre la desigualdad que se evidencian en ellos.

Metodológicamente, hemos efectuado el análisis bibliográfico sobre producciones teóricas que conceptualizan la desigualdad social, particularmente desde la sociología. Ello implico análisis de fuentes primarias y secundarias al respecto. Para el estudio de antecedentes sobre políticas estatales destinadas al tratamiento de la desigualdad social hemos construido un criterio de selección que combine especificidad temática y de objeto, alcance de las políticas seleccionadas y diversidad de enfoque teórico. Para luego, cruzar y hacer dialogar producciones específicamente teóricas con aquellas de contenido y análisis empírico.

\section{Los ciclos de las políticas sociales y educativas en Argentina}

Desde finales del siglo pasado hasta la actualidad han cobrado gran relevancia diversos proyectos y programas destinados a poblaciones en situaciones de vulnerabilidad social que ven afectados derechos elementales consagrados en tratados, convenciones y leyes nacionales e internacionales como es el derecho a la educación. Esto da cuenta de un tipo de intervención que los Estados nacionales Latinoamericanos llevaron y llevan adelante ante la problemática de la exclusión y desigualdad social en la región convirtiendo demandas emanadas de diversos sectores y actores sociales en un tipo de política pública que, según el país y los períodos de gobierno, han significado de distintas maneras.

Este tipo de intervención estatal no inicia en las últimas décadas del siglo XX, sin embargo es a partir de este particular momento histórico donde se expresa y cristaliza en la institucionalidad Estatal un tipo de política destinada a promover el acceso a la educación, la igualdad de oportunidades, el mejoramiento en los aprendizajes, reducir la deserción y el abandono escolar de amplios sectores sociales que en esas décadas fueron afectados por las políticas de orientación neoliberal (Duschatzky y Redondo, $2000 a$ y 2000b; Feldman, Atorresi y Merkel, 2013). 
En estas dos últimas décadas del siglo XX, toda la región lleva adelante una serie de reformas educativas (y no solo educativas) impulsadas por organismos multilaterales que tienen un fuerte componente eficientista donde en cada país implicó reformas profundas, pero también diversas (Tiramonti y Suasnabar, 2000). Particularmente, en Argentina, este proceso se cristaliza en los años noventa con la sanción, primero, de la Ley de Transferencia Nro. 24.0496 en el año 1992, y, luego, con la Ley Federal de Educación (LFE) Nro. $24.195^{7}$ sancionada en 1994. Entre ambas leyes, en el año 1993, el Plan Social Educativo (PSE) se convierte en el antecedente fundamental de este tipo de política destinada a los sectores vulnerables que combina la asistencia social y educativa.

En la década siguiente, luego de la profunda crisis económica, social, política e institucional que estalla en los finales del año 2001, se retoma un clima de reformas donde las educativas vuelven a tener un papel importante (Feldfeber y Gluz, 2011). En el año 2006 se sanciona la Ley de Educación Nacional (LNE) Nro. 26.206 en un clima y con una impronta de restitución del derecho a la educación en tanto bien social y con el Estado como actor central en garantizar y hacer efectivo el derecho a enseñar y aprender de todos y todas las ciudadanas.

Dos años antes, en 2004, lo que era el PSE también es afectado como política de Estado y se lanza el Plan Nacional de Inclusión Educativa (PNIE) buscando dar nuevas respuestas a la problemática de la exclusión escolar y la desigualdad social. Así, el PNIE junto a otras iniciativas como la creación

\footnotetext{
6 Esta ley transfirió la administración de los servicios educativos que aún dependían del Ministerio de Educación y Cultura de la Nación hacia las jurisdicciones provinciales y de la Ciudad de Buenos Aires. Cabe aclarar que la misma completa un proceso iniciado por la dictadura de los años 1966-1973, revertido en el período democrático 1973-1976 y ejecutado en la dictadura militar de los años 1976-1983 (Braslavsky, 1986) que en el año 1978 sanciono la Ley 21.809 de "Transferencias de servicios educativos a provincias" transfiriendo la administración de las escuelas preprimarias y primarias dependientes del Consejo Nacional de Educación a las jurisdicciones provinciales.

7 Ley que regulo el sistema educativo argentino hasta el año 2006 cuando se aprobó la Ley de Educación Nacional, nro. 26.206.
} 
de la Dirección Nacional de Políticas Socioeducativas (DNPS) 8 marcan una nueva manera en la que el Estado va a intervenir en los asuntos públicos y en particular con la problemática de la desigualdad social y educativa (Monath, 2016). Tanto el PNIE como, más adelante, la DNPS definen la manera de tramitar las desigualdades sociales y educativas y los efectos de la exclusión desde el mandato de la igualdad y la calidad educativa. La orientación de la política pública es redefinida incorporando sentidos de pretensiones universalistas e igualitaristas. Se deja el mandato de la equidad, la asistencia y la carencia que expresó el reformismo de los 90 por el mandato de la igualdad, la calidad y la inclusión educativa (Senén González, 2008; Senén González y Olmos, 2014; Monath, 2016). En este sentido, surgen toda una serie de programas y proyectos denominados socioeducativos que buscarán abordar la problemática de la desigualdad social y educativa desde aquellos mandatos y aquellas pretensiones políticas.

Se da, entonces, una reconfiguración de las políticas sociales y educativas reorientadas por los mandatos que cada país en la región imprimió a su modo de identificar, gestionar e intervenir en los asuntos públicos (Saforcada y Vassiliades, 2011). En Argentina, las políticas compensatorias que tenían como mandatos la calidad y equidad educativa y nombraban a la población destino como carente pasaron a denominarse socioeducativas e incorporaron los mandatos de la igualdad y calidad educativa plasmados en la LEN designando a los destinatarios de estas políticas como sujetos de derechos (Monath, 2016). Junto a la idea de igualdad y calidad aparece fuertemente el mandato de la inclusión social, en tanto las políticas sociales y educativas debían promover la inclusión de todos aquellos y aquellas que vieron afectado el cumplimiento pleno del derecho a la educación que la nueva legislación establecía en su novedad, la obligatoriedad del nivel secundario. De este modo, el sistema educativo

\footnotetext{
8 En el año 2010 se crea bajo la órbita de la Subsecretaría de Equidad y Calidad del Ministerio de Educación de la Nación la Dirección Nacional de Políticas Socioeducativas.
}

Revista Exitus, Santarém/PA, Vol. 10, p. 01-30, e020061, 2020. 
argentino se conformaba en obligatorio desde el nivel inicial al nivel secundario, quedando exceptuado el nivel superior.

Así, las políticas socioeducativas se constituyeron como un tipo de intervención estatal sobre aquella población en situación de vulnerabilidad social, con una impronta de igualdad y calidad educativa, y de reposición de derechos, a la vez que busco la inclusión de estos sectores al sistema educativo.

\section{Antecedentes sobre las políticas compensatorias y socioeducativas en}

\section{Argentina}

Entre los antecedentes de investigación relevados encontramos la tesis de maestría de la autora brasilera Gladys Barreyro (2001). Su objeto de análisis lo constituye el Plan Social Educativo (PSE) sancionado, como se mencionó, en el año 1993 bajo el primer mandato del presidente Menem. Para la autora, esta política implementada fue el gran antecedente en el que se incorporó la "cuestión social" al ámbito de las políticas educativas. La población destinataria a la que el Estado convirtió en beneficiaria se la identificó y definió en tanto "pobres", "necesitados" y "postergados", enfatizando una función de asistencia por sobre la función de garante y sostén de derechos que caracterizó al sistema educativo argentino.

Una de las cuestiones interesantes que la autora destaca refiere a que la iniciativa e implementación del PSE tuvo que ver no solo con una política de reducción del déficit fiscal que llevaba adelante el gobierno de entonces, sino también con una política que busco contener la conflictividad social en alza destinando una gran cantidad de recursos para tales fines. Con ello, la política del PSE se ubicó en el delicado equilibrio entre ajuste y gasto social selectivo (Barreyro, 2001).

En lo pedagógico el PSE intentó llevar adelante los valores de mercado a la esfera educativa. La autora los identifica en la autonomía asignada a las escuelas, la focalización de la intervención y provisión de recursos, el intento 
de instalar la competencia entre instituciones para la obtención de recursos, la inversión selectiva y la descentralización. De modo que asistimos no solo a un modo de gestionar y administrar recursos estatales que, como muestra la autora, están fuertemente orientados por la necesidad de ajustar los gastos del Estado sino también a la producción de sentidos y valores propios del modelo de acumulación neoliberal, esto es: "un Estado que ajustaba, pero se ocupaba de los pobres, de modo tutelar" (2001, p.177).

El otro antecedente fundamental que aquí destacamos refiere al estudio que llevaron adelante Duschatzky et. al. (2000a, 2000b) sobre el Plan Social Educativo. Las autoras buscan "interrogar al PSE inscripto en una cadena significante" (2000b, p.3), es decir, en la discursividad reformista de fin de siglo. A partir de esta referencia teórica su análisis nos permite advertir que el PSE fue mucho más que el modo en que el Estado administro y gestiono recursos para una porción importante de la sociedad, sino que su implementación marca la ruptura simbólica del horizonte universal e igualador que caracterizó al sistema educativo argentino desde su surgimiento.

Así como para Barreyro (2001) el PSE es la incorporación de "lo social" al campo de las políticas educativas, para Duschatzky et. al. (2000a y 2000b) esta política inaugura una nueva modalidad de inclusión educativa donde se polarizan los beneficiarios de las políticas educativas en sujetos necesitados y sujetos competentes, quebrando la utopía del horizonte integrador que caracterizó al sistema educativo argentino desde su surgimiento. De este modo, el "PSE convalida la ruptura de procesos de integración educativa y de identidades colectivas históricamente configuradas" (2000b, p.3). Se configuran nuevas identidades, nuevos mecanismos de interpelación por parte del Estado hacia su población. Ya no serán los ciudadanos de derechos, soberanos los que la política educativa constituye, sino que habrá sujetos competentes y sujetos de asistencia. Los sujetos a los que el PSE focaliza su atención son "pobres", "carenciados", "necesitados", "vulnerables" que merecen su asistencia. 
Por otro lado, las autoras desenmascaran la concepción de pobreza que lleva consigo la reforma educativa de los 90 y el PSE como el instrumento para tramitarla. De cierto modo, como se dijo, objetivando sujetos de carencia y necesidad. Lxs pobres no se encuentran en situación de pobreza producto de un tipo (desigual) de distribución de las riquezas, ni siquiera son sujetos de derechos, sino sujetos a asistir. Por otro, destacando que la pobreza se tramitó vía focalización, es decir restringiendo el problema a una porción de lo social y no a su relación compleja con otras dimensiones de producción de la desigualdad. De esta manera, las autoras afirman que el PSE trae consigo una concepción de individualización de la pobreza y de reducción de los problemas que ella demanda a cuestiones distribucionistas (Duschatzky et. al., 2000b).

A través del trabajo de las autoras mencionadas se puede dar cuenta que el problema de PSE, o la crítica posible, no refiere meramente a la cuestión de la administración de recursos por parte del Estado. En palabras de ellas, "del orden de la prestación", sino que lo que distingue "a los destinatarios de la educación en general, de los incluidos en la esfera del PSE es la naturaleza de la relación construida con el estado, es la posición en que son situados" (2000b, p.18). Una posición en la que los sujetos quedan desprovistos de derechos, su posición de carentes y necesitados sólo los habilita a pedir ayuda y en la misma operación reconocerse como tales. Un Estado que no universaliza derechos, que no crea ciudadanía, que no educa igual para todos, sino que fractura y fragmenta su vínculo con la ella.

El tercer antecedente corresponde al artículo de la autora Oreja Cerruti (2016) en el que se presentan las conclusiones de una investigación que centró su análisis en las acciones del Ministerio de Educación Nacional a través de la implementación de diversos programas para la educación obligatoria orientados a la calidad, igualdad, inclusión y equidad en el período 2003-2015.

En este trabajo se considera que más allá de los cambios discursivos que se plasmaron en el ámbito de las políticas educativas, particularmente 
de las socioeducativas en Argentina poscrisis 2001, la implementación de los programas estudiados "constituyeron acciones flexibles, fragmentadas y parciales respecto de los objetivos declarados" (2016, p. 159), en relación a sus objetivos explícitos. Con ello, se contribuyó "a reforzar la apariencia del Estado nacional como árbitro entre clases sociales y a la construcción de consenso" (2016, p. 160). En este sentido, para Oreja Cerruti "la cuestión no estriba tan sólo en plantear si el Estado dio respuesta o no a un problema, sino en caracterizar y evaluar el tipo de respuesta y su papel respecto de los intereses antagónicos de las clases sociales" (2016, p. 154).

Así, los programas y políticas públicas estudiadas aquí fueron analizados en relación a si afectaron o no intereses de clase. A partir de aquí se consideró que todos los programas y acciones del ministerio de educación en el período 2003-2015 evidencian una continuidad con los lineamientos de los organismos internacionales $y$, por otro lado, que estos programas mostraron una serie de características propias al nuevo modelo del capitalismo: la flexibilidad se la asocia al management y neotoyotismo, la forma de trabajo a la precarización laboral y los vínculos que habilita la política socioeducativa con organizaciones civiles obedeció a "oculta[r] los antagonismos de clases" (p. 159). Con todo lo expresado, esta investigación ubica a las políticas denominadas socioeducativas en continuidad con las de la década anterior, denominadas compensatorias, estableciendo que la diferencia radicó en la manera en que el Estado enunció tales acciones.

Por último, un cuarto antecedente lo constituye un estudio en el campo de las políticas públicas, particularmente en aquellas que son nuestro objeto de análisis, con interés en algunas dimensiones propias del campo de la Educación Física. Nos referimos al trabajo de Aisenstein et. al. (2011). El mismo se propuso analizar programas sociales, educativos y de salud ${ }^{9}$ del

\footnotetext{
9 Los programas seleccionados por las autoras fueron: Escuelas abiertas de verano, Patios abiertos y Escuelas deportivas de la Dirección General de Cultura y Educación de la provincia de Buenos Aires.
} 
ámbito de la política pública jurisdiccional 10 que incorporaron aquellos contenidos propios de la cultura física.

En su análisis de los programas relevados las autoras elaboran una tipología delimitando, por un lado, programas que conceptualizan a las prácticas deportivas y recreativas como productoras de capital social y cultural, considerando que su acceso permitiría brindar mayores herramientas a los sujetos destinatarios. Por otro lado, aquellos programas que significan a las prácticas corporales que ofrecen como bienes sociales, otorgando valor a la práctica corporal en sí misma y que, por lo tanto, la virtud radicaría en el acceso de la población en situación de desigualdad a estos bienes sociales negados.

En esta tipología las autoras afirman que el tipo de intervención que llevaban adelante profesores y profesoras de EF eran, para el primer tipo, centralmente lúdicas y recreativas sin un tipo de intervención orientada a la enseñanza, más bien predominando la puesta a disposición de prácticas corporales. En cambio, para el segundo tipo, la forma que adoptaba la intervención de docentes era el referencial deportivo donde la enseñanza de aspectos del deporte orientaba la propuesta del programa.

Algo que destacan Aisenstein et. al. (2011) es que en este tipo de programas las representaciones e intervenciones de profesores y profesoras de EF se encuentran tensionadas entre la enseñanza y la recreación, es decir en el énfasis que estos profesores/as otorgan a sus prácticas específicas: si enseñan algún contenido o recorte cultural de una práctica corporal o si prevalece la experiencia lúdica-deportiva de sus destinatarios. Según las autoras, esta tensión se observa con mayor claridad en la primera tipología.

Por otro lado, resulta relevante destacar, a los fines de la investigación en curso, la descripción que las autoras caracterizan sobre la tipología de docente que prevalece. Es interesante en tanto nuestra investigación indagará sobre la construcción de un tipo de posición docente que asumen

10 Refiere a la administración provincial. Para el caso en cuestión de la provincia de Buenos Aires.

Revista Exitus, Santarém/PA, Vol. 10, p. 01-30, e020061, 2020. 
profesores y profesoras de EF en programas socioeducativos. En este caso, observan que prevalece un tipo de docente con "rasgos o cualidades de algunos modelos emancipatorios de la educación física humanista de los años '70 en el sentido que el contexto político, cultural y social, estaría determinando diariamente la selección y organización de sus propuestas" (2011: 1). Al mismo tiempo sostienen, con Fenstermacher \& Soltis (1998 citado en Aisenstein, 2011), que ese modelo coexiste con un modelo de docente terapeuta más orientado a potenciar el crecimiento personal e individual y la autoafirmación de cada persona.

Finalmente, el trabajo también aborda cuestiones relativas propias a este tipo de políticas públicas destinadas a reducir situaciones de desigualdad. En ese sentido dejan planteado el interrogante por cómo es posible trabajar en pos de aquel objetivo cuando se trata de intervenciones que no se centran en la transferencia de recursos y servicios. Digamos, hay "algo más" que allí se juega (la apropiación de un elemento, práctica, saber cultural) y que no es posible cuantificar y certificar. Con esto, las autoras plantean que la cuestión de la desigualdad no es un problema sólo económico, sino que existen varios elementos que producen desigualdad(es). Entendida esta multidimensionalidad de la desigualdad se sugiere en el trabajo que un abordaje parcial de la desigualdad no permite resolver su producción.

\section{Perspectivas sociológicas en estudios sobre políticas sociales y educativas: Conclusiones provisorias}

A partir de lo esbozado hasta aquí y retomando las conceptualizaciones sobre desigualdad que se desarrollaron al comienzo del trabajo podemos identificar las perspectivas o enfoques teóricos con el que los distintos trabajos han interrogado las políticas destinadas al problema de la desigualdad social y educativa. 
Retomando la caracterización de Reygadas (2004) sobre las teorías que abordan la desigualdad social, los trabajos de Duschatzky et. al. (2000a, 2000b) y de Barreyro (2001), lo hacen desde un enfoque interaccionista, en la medida que centran su atención en un programa y política puntual y desde allí evidencian los mecanismos político-simbólicos de producción de desigualdades que se despliegan en la institucionalidad estatal. Ambas recuperan y presentan elementos de teorías holísticas o macro estructurales, ya que presentan a su objeto de estudio, el Plan Social Educativo, como parte de una nueva fase de capitalismo mundial: globalización, liberalización, privatización, reconfiguración de los Estados.

Sin embargo, ambos estudios permiten ver algo más que esa tendencia global de transnacionalización y liberalización económica. Ellas nos permiten ver qué características adoptó en Argentina y, más particularmente, cómo impacto ese proceso de reformas en las políticas educativas argentinas que, a diferencia de otros países de la región, habían tenido una impronta fuertemente centralista, igualitarista, inclusiva y de movilidad social ${ }^{11}$. Con ello, nos muestran que el PSE se trató no solo de un ajuste fiscal y de reorientación del Estado en términos de sus funciones y delegaciones, sino que implico un cambio en la matriz cultural y simbólica de las políticas de Estado en relación a la ciudadanía, produciendo efectos en la construcción de la misma: se convalido una ciudadanía competente y una ciudadanía carente. Los primeros fueron interpelados no por sus derechos sino por sus capacidades de competir en el libre mercado. Los otros fueron convocados por sus necesidades y sus carencias, naturalizando su condición desigual y produciendo un ciudadano a asistir y a compensar. Ello produjo una estigmatización de los ciudadanos "necesitados" y las escuelas pasaron a cumplir funciones de asistencia por sobre de enseñanza convalidando identidades fragmentadas.

\footnotetext{
11 Son conocidas las críticas al proceso de homogeneización e imposición cultural que supuso el sistema educativo argentino. De ello, las autoras Duschatsky y Redondo (2000a y 2000b) dan cuenta del proceso de homogeneización cultural que implico la escuela moderna argentina como así al mismo tiempo su carácter inclusivo y democrático.
} 
Las conclusiones que se expresan dan cuenta de un abordaje de tipo interaccionista. Si se hubiera optado por una dimensión de tipo macro estructural (Reygadas, 2004), u objetiva de la desigualdad (Saraví, 2015), probablemente sólo se hubiera podido captar cierta continuidad de la dinámica mundial de reformas neoliberales en la nueva legislación educativa argentina. Ello nos permitiría dar cuenta de las reconfiguraciones del Estado y sus funciones, el ajuste fiscal y su impacto social, la novedosa aparición y combinación de lo social y lo educativo en las políticas educativas, pero dejaría cierta vacancia en aquellas estrategias políticosimbólicas que intervienen en la producción de desigualdades (Reygadas, 2004) como de su carácter relacional y multidimensional (Saraví, 2015). Los trabajos de Barreyro (2001) y Duschatzky et. al. (2000a y 2000b) identifican muy claramente cómo el PSE ha sido el instrumento que contribuyó a preservar las desigualdades sociales entre grupos convalidando circuitos de asistencia y carencia y circuitos competentes. Mediante la focalización y asignación de recursos situó el problema de la desigualdad social en el orden de la prestación y no en los mecanismos de reproducción de las desigualdades. Eso es posible vislumbrarlo en el modo en que el Estado nombro e invistió a las escuelas y población destinataria. Al convocarlos desde su necesidad, las escuelas e individuos se convirtieron en ciudadanos de asistencia y de ayuda y no como ciudadanos plenos.

Continuando con los enfoques sociológicos que destacan la multidimensionalidad de la(s) desigualdad(es) social(es) (ahora en plural), los trabajos de las autoras nos permiten observar la producción de inclusiones diferenciadas y desiguales (Saraví, 2015), en tanto, la política del PSE y las reformas educativas llevadas adelante en la última década del siglo XX, produjeron acumulación de desventajas por parte de los sectores empobrecidos, convalidaron circuitos diferenciales $y$, advirtiendo el factor relacional que Saraví (2015) destaca de todos los procesos sociales, consolidaron circuitos de inclusión privilegiada en aquellos grupos sociales acomodados. 
En cambio, el estudio sobre los programas llevados adelante por el Ministerio de Educación en Argentina en el período 2003-2015 de Oreja Cerruti (2016), podemos situarlo más cerca de los enfoques de tipo macro estructurales o con énfasis en la dimensión económico-estructural ya que, al decir de la autora, se ha "buscado superar un enfoque meramente técnico o normativo que 'midiera la eficiencia' de los programas" (p. 142), contraponiendo aquellos enfoques que encuentran una conexión fundamental entre la dimensión estatal y la estructura de una formación social, capitalista. Como se observa, se adscribe de manera explícita a una perspectiva que con Reygadas (2004) caracterizamos como holísticas, es decir, aquellas que enfatizan las dimensiones macro estructurales de lo social, las dinámicas transnacionales y procesos a gran escala del actual modelo de acumulación.

Siguiendo esta perspectiva la autora encuentra continuidades de los programas estudiados con las líneas propuestas a nivel mundial por los organismos multilaterales de crédito (UNICEF, Banco Mundial, UNESCO), tanto en lo discursivo como en su implementación y orientación. También se considera que las lógicas de los programas en su implementación se relacionan a las nuevas lógicas de trabajo y relación social que impone la dinámica económica (neotoyotismo, management). Se afirma en este trabajo que el Estado sólo ha cambiado lo discursivo: la forma de nombrar la desigualdad/igualdad, la educación, el derecho, en sus palabras, "la apariencia", pero no ha podido intervenir en la lucha de clases o, mejor, no ha afectado los intereses de las clases dominantes.

A nuestro entender, la perspectiva que asume el trabajo de Oreja (2016) permite arrojar luz sobre aquellos procesos de tendencia global que han tenido gran impacto en las reformas educativas de la región a partir de la última década del siglo XX, como fueron la conversión del bien educación como un servicio y una mercancía más a adquirir en el mercado, la desregulación y descentralización de los sistema educativos nacionales por parte de los Estados nacionales hacia administraciones jurisdiccionales y 
municipales y también privadas y de organizaciones no gubernamentales, la tendencia hacia la formación de recursos humanos para un mercado globalizado, la participación e influencia de los organismos multilaterales de créditos en la orientación y financiamiento de esas reformas, entre otros procesos. Sin embargo, el trabajo encuentra una serie de problemas para el análisis de los programas del período en cuestión que queremos destacar:

El primero es de enfoque. Si se pretende analizar las concepciones político-educacionales de los programas, su impacto en la calidad de los procesos que despliegan y sus logros en relación a los objetivos que declaran, una perspectiva centrada únicamente en el análisis de los grandes actores y procesos macro económicos descuida o no permite dirigir la mirada a los procesos de resignificación, reorientación, movilización de recursos, negociación y resistencias que toda política de Estado supone. Sabemos con Oszlak que "Ias políticas estatales se insertan en una 'estructura de arenas" (1981, p. 5) y que es necesario conocer para entender cómo se resuelven ciertas cuestiones 12 y no otras, y cómo las tramita el Estado en su toma de posición. Así, una perspectiva que enfatiza la tendencia global en la influencia local pierde potencia para el análisis de los procesos locales.

El segundo refiere a la concepción teórica de aquel enfoque. De todas las dimensiones que se juegan en los procesos sociales globales la autora pondera el de la estructura de clases sociales. Ella redunda en una concepción economicista de las políticas estatales y, particularmente de las educativas, que asocia todas sus dimensiones a un carácter último de clase, ignorando la autonomía de lo político y lo ideológico (Mouffe, 1980). Esto es notorio cuando se afirma que es necesario evaluar esos programas que promovió el Estado en relación a si afecto o no los interesas antagónicos de las clases sociales (Oreja, 2016). Como consecuencia de esta postura teórica

\footnotetext{
12 Sobre las cuestiones Oszlak sostiene que "ninguna sociedad posee la capacidad ni los recursos para atender omnímodamente a la lista de necesidades y demandas de sus integrantes. Sólo algunas son 'problematizadas', en el sentido de que ciertas clases, fracciones de clase, organizaciones, grupos o incluso individuos estratégicamente situados creen que puede y debe hacerse 'algo' a su respecto y están en condiciones de promover su incorporación a la agenda de problemas socialmente vigentes. Llamamos 'cuestiones' a estos asuntos (necesidades, demandas) 'socialmente problematizados" (1981, p. 12).
} 
es de esperar que las concepciones pedagógicas e ideológicas de las políticas socioeducativas reproduzcan concepciones de clase.

El tercero, finalmente, lo planteamos con el trabajo de Aiseinstein et al. (2011) cuando se preguntan por cómo cuantificar, evaluar y certificar procesos de reducción de desigualdades educativas en programas que no se agotan en la transferencia de recursos materiales y de servicio. Es evidente que esta preocupación no es de interés del trabajo de Oreja (2016), sin embargo, creemos que aquel trabajo nos permite analizar otros componentes de la política en cuestión que impactan en el mantenimiento, reducción, reproducción, tratamiento de las desigualdades sociales, a saber: cómo son presentados y abordados las propuestas educativas y culturales tanto por los programas como en los actores intervinientes; el tipo de posicionamientos político pedagógicos en relación a la igualdad/desigualdad que asumen los maestros y maestras de los programas; las tensiones entre la enseñanza y la recreación, la asistencia social y la garantía de derechos; la apropiación de los destinatarios de los programas de recursos materiales y simbólicos para mantener o incrementar posiciones sociales; las estrategias de los actores institucionales; entre otras dimensiones.

Hasta aquí hemos podido recuperar brevemente las principales teorías y perspectivas sobre la desigualdad social para analizar una serie de trabajos sobre políticas públicas destinadas al tratamiento de la desigualdad social en Argentina que constituyen los antecedentes de un proyecto de investigación en curso. Esto nos permitió incorporar al análisis el aspecto relacional y multidimensional de la desigualdad social, por un lado, e interrogar aquellos trabajos en sus aspectos conceptuales, metodológicos y conclusivos, por otro. Así, hemos podido dar cuenta de trabajos anclados en una perspectiva macroestructural con énfasis en la dimensión económica de la desigualdad social y otros que incorporan la dimensión macro estructural, pero enfatizan el nivel de las interacciones institucionales y mediaciones locales. 
Con todo, el presente artículo evidencia cambios en las perspectivas teóricas sobre el abordaje de la desigualdad social que se verifican en las cuatro investigaciones analizadas. Cada una permite conclusiones en relación a sus postulados teóricos y objetos construidos. Sin embargo, para el análisis de políticas públicas que combinan el aspecto social con la dimensión pedagógica y educativa, hemos observado que esos estudios merecen un abordaje multidimensional que escapen a los determinismos económicos o culturales. Así lo evidenciamos en el análisis de aquellas investigaciones que estudiaron este tipo de políticas públicas en el período seleccionado y que aquí caracterizamos como de surgimiento de un nuevo modo en que los Estados, particularmente el argentino, tramitaron la(s) desigualdad(es) social(es).

\section{REFERENCIAS}

Aisenstein, Á., Ganz, N., Perczyk, J., Benito, F., Bamonte, L. y Baioni, G. (2011). Aspectos de la cultura física en los programas sociales, educativos y de salud. Educación Física y Deportes, año 15, n. 152, enero 2015. Recuperado el 11 de mayo de 2020, de https://www.efdeportes.com/efd152/aspectosde-la-cultura-fisica-en-los-programas-sociales.htm

Barreyro, G. (2001) Políticas educativas en la Argentina a fines del siglo XX: un estudio del Plan Social Educativo. Tesis de Maestría. Facultad Latinoamericana de Ciencias Sociales.

Bendix, R. (1974). Transformaciones experimentadas por las sociedades de Europa occidental a partir del siglo XVIII. En: Estado Nacional y Ciudadanía. Buenos Aires: Amorrortu.

Braslavsky, C. (1986). La discriminación educativa en la Argentina. Buenos Aires: Miño y Dávila.

Carriquiriborde, N. (2019). La perspectiva sociológica en bourdieu: intersecciones entre el campo socioeducativo y el campo de la educación física. Revista Tempos E Espaços Em Educação, 12 (29), 227-244. 
Dubet, F. (2011). Repensar la justicia social. - $1^{a}$ ed.- Buenos Aires: Siglo XXI Editores.

Dubet, F. (2018). Los desafíos de la justicia escolar. Ciudadanías. Revista de Políticas Sociales Urbanas N³, 2do semestre 2018, 7-24.

Duschatzky, S. y Redondo, P. (2000). Las marcas del Plan Social Educativo o los indicios de ruptura de las políticas públicas. En Duschatzky, S. (comp.) Tutelados y Asistidos. Programas sociales, políticas públicas y subjetividad. Buenos Aires: Paidós.

Duschatzky, S. y Redondo, P. (2000b). El Plan Social Educativo y la crisis de la educación pública. Reflexiones sobre los sentidos de las políticas compensatorias en los tiempos de la Reforma Educativa. Buenos Aires, FLACSO.

Feldfeber, M. \& Gluz, N. (2011). Las políticas educativas en la Argentina: herencias de los '90, contradicciones y tendencias de 'nuevo signo'. Educação \& Sociedade, Campinas, v. 32, n. 115, 339-356, abr.-jun.

Feldman, D., Atorresi, A. y Mekler, V. (2013) Planes y programas para mejorar el aprendizaje y reducir el fracaso en la educación básica en América Latina. Revista Latinoamericana de Educación Comparada, año 4/ nro. 4. Buenos Aires, SAECE.

Hobsbawn, Eric (1964). La carrera abierta al talento. En: La revolución burguesa. Madrid: Guadarrama.

Mora Salas, Minor (2004). Desigualdad social en América Latina: viejos problemas, nuevos debates. Cuaderno de Ciencias Sociales. no. 131. San José: FLACSO.

Monath, H. (2016). Tensiones y diálogos entre políticas sociales y políticas educativas en el diseño de programas de inclusión en la escuela secundaria argentina (1990-2010). Tesis de Maestría. Facultad Latinoamericana de Ciencias Sociales.

Mouffe, C. (1980) Hegemonía e ideología en Gramsci. En teoría, 5, 115-156. 
Oreja Cerruti, M. B. (2016). Forma y contenido de la política educacional: Ios programas del Ministerio de Educación Nacional para el mejoramiento de la calidad, la equidad y la inclusión (2003-2015). Polifonías Revista de Educación, Año V, № 8, 140-168

Oszlak, O. y O’Donnell, G. (1981). Estado y políticas estatales en América Latina: hacia una estrategia de investigación. Centro de Estudios de Estado y Sociedad (CEDES), Documento G.E. CLACSO. Vol. 4. Buenos Aires, Argentina. Reygadas, L. (2004). Las redes de la desigualdad: un enfoque multidimensional. Política y Cultura, n²2, 7-25.

Saforcada, F. y Vassiliades, A. (2011). Las leyes de educación en los comienzos del siglo XXI: del neoliberalismo al postconsenso de Washington en América del Sur. Educação \& Sociedade, Campinas, v. 32, n. 115, 287-304, abr.jun. 2011.

Saraví, G. A. (2015). Juventudes fragmentadas: socialización, clase y cultura en la construcción de la desigualdad. México: Flacso México: CIESAS.

Senén González, S. (2008). Política, leyes y educación. Entre la regulación y los desafíos de la macro y la micro política. En Perazza, R. (comp.). Pensar en lo público: notas sobre la educación y el estado. Buenos Aires: Aiquen grupo. Senén González, S. y Olmos, A. (2014). De las políticas compensatorias a la ampliación de derechos: una década de proyectos y programas socioeducativos en la Argentina y en Córdoba. Cuadernos de Educación. Año XII - No 12 - noviembre 2014 1-13.

Southwell, M. (2017). El Análisis Político del Discurso y la cuestión de la politicidad de la educación. En Padierna Jiménez, M. y García Contreras M. (coord.). Programa de Análisis Político de Discurso e Investigación (pp. 109131). México, D.F.: Plaza y Valdés.

Southwell, M.; Vassiliades, A. (2014). El concepto de posición docente: notas conceptuales y metodológicas. Educación, Lenguaje y Sociedad ISSN 16684753 Vol. XI N 11, 163-187.

Tiramonti, G. y Suasnabar, C. (2000). La reforma Educativa Nacional en busca de una interpretación. Aportes para el Estado y la Administración Gubernamental. Año 7, Número 15, Otoño 2000. Bs. As. 
Recebido em: 18 de maio de 2020 Aprovado em: 02 de agosto de 2020 Publicado em: 03 de agosto de 2020 (c) (1) 8 